\section{Back under a \\ SQL Umbrella}

ADRIAN COLYER
UNIFYING SERVING

AND ANALYTICAL DATA

WITH PROCELLA;

USING A DATABASE

FOR DISTRIBUTED

MACHINE LEARNING

6ad the great privilege of being able to attend VLDB 2019 in person this year. The conference was packed with interesting people, posters, and presentations and I was made to feel very welcome by everyone I met. If you're a practitioner wondering whether or not an academic conference is for you, I can highly recommend it. I've chosen two papers from the conference for this edition, out of many strong candidates.

Procella is the latest in a long line of data processing systems at Google. What's unique about it is that it's a single store handling reporting, embedded statistics, time series, and ad-hoc analysis workloads under one roof. It's SQL on top, cloud-native underneath, and it's serving billions of queries per day over tens of petabytes of data.

There's one big data use case that Procella isn't handling today though, and that's machine learning. But in 'Declarative recursive computation on an RDBMS... or, why you should use a database for distributed machine learning,' Jankov et al. make the case for the database being the ideal place to handle the most demanding of distributed machine learning workloads.

Could everything be coming back together again under a SQL umbrella?

For the complete column, go to https:/lqueue.acm.orgl TheMorningPaper/5/ 
Adrian Colyer is a venture partner with Accel in London, where his job is to help find and build great technology companies across Europe and Israel. IIf you're working on an interesting technology-related business, he would love to hear from you: you can reach him at acolyer@accel.com.] Prior to joining Accel, he spent more than 20 years in technical roles, including CTO at Pivotal, VMware, and SpringSource.

Copyright $@ 2019$ held by ownerlauthor. Publication rights licensed to ACM. 